\title{
A Commentary on The importance of knowing from whence your PHOX2B mutation comes
}

\author{
Casey M Rand ${ }^{1}$ and Debra E Weese-Mayer ${ }^{1,2}$ \\ Journal of Human Genetics (2012) 57, 345-346; doi:10.1038/jhg.2012.44; published online 3 May 2012
}

$\mathrm{M}$ eguro et al. ${ }^{1}$ have more fully characterized the growing cohort of patients with Congenital Central Hypoventilation syndrome (CCHS) in Japan. Specifically, they have confirmed the autosomal dominant inheritance pattern ${ }^{2,3}$ and described the incidence of somatic mosaicism or full mutation in parents of CCHS probands in the Japanese cohort (22\%)-reflecting similar figures to the report (using the same methodology) as Bachetti et al. ${ }^{4}$

These results raise several important points in regard to genetic and physiologic testing procedures in CCHS families. First, the results emphasize the importance of genetic counseling for families in which a CCHScausing $P H O X 2 B$ mutation is identified. This report, and others, has identified families in which recurrence of these $P H O X 2 B$ mutations and this disorder has occurred; typically an asymptomatic parent carried somatic mosaicism for a mutation, and without genetic counseling and proper genetic testing these families were without valuable information to advise family planning. Second, this report underscores the necessity of physicians to recommend and to complete thorough clinical and physiological evaluations to assure safety in these families. Here, Meguro et al. identified a family in which, after presentation of a single proband with a 25-alanine expansion mutation in $\mathrm{PHOX} 2 \mathrm{~B}$, familial testing revealed the same mutation in two previous generations of the proband's family. After this discovery, polysomnography revealed symptoms of late-onset CCHS

Casey M Rand and Debra E Weese-Mayer are at the ${ }^{1}$ Children's Memorial Hospital, Center for Autonomic Medicine in Pediatrics, Chicago, IL, USA and ${ }^{2}$ Department of Pediatrics, Northwestern University Feinberg School of Medicine, Chicago, IL, USA.

E-mail: CRand@ChildrensMemorial.org in each of these mutation carriers, both previously considered asymptomatic. Third, these results reiterate the need to carefully consider the mechanism chosen for $\mathrm{PHOX} 2 \mathrm{~B}$ testing among probands and among parents of probands in order to optimize management, assure safety and physiologic stability, and to determine risk of recurrence in subsequent offspring. This report supports previous findings ${ }^{3-5}$ indicating inheritance of $P H O X 2 B$ mutations from parental carriers with very low levels of somatic mosaicism (as low as $5 \%$ here). Although sequencing of the $P H O X 2 B$ gene has become the most commonly available form of clinical testing in CCHS families, studies have shown it to be inadequate to identify low-level mosaicism. ${ }^{4,5}$ Without proper testing procedures, no amount of clinical acumen or informed genetic counseling will be able to correctly inform families of recurrence risk. Figure 1 provides the algorithm to determine when and what type of $P H O X 2 B$ genetic testing should be performed in parents of a CCHS proband. Though the American Thoracic Society (ATS) Statement on CCHS published in $2010^{6}$ clearly recommended parental testing, it has become apparent that physicians are not consistently recommending parent testing for $P H O X 2 B$ mutations, and

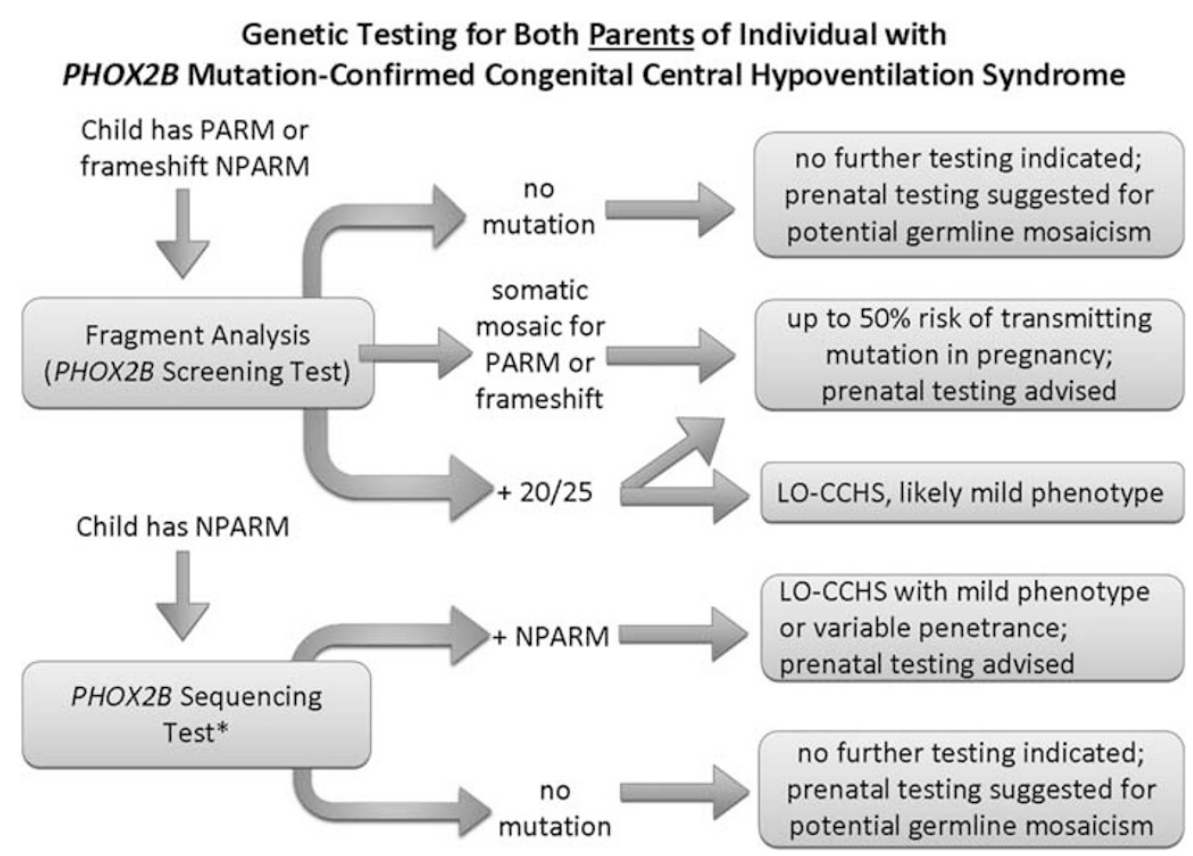

Figure 1 Algorithm to determine when and what type of $P H O X 2 B$ genetic testing should be performed in parents of $\mathrm{CCHS}$ probands. PARM, polyalanine repeat expansion mutation; NPARM, non-polyalanine repeat expansion mutation. Reproduced with permission from Weese-Mayer et al. ${ }^{7}$ A full color version of this figure is available at the Journal of Human Genetics journal online. 
they are not necessarily recommending the correct type of testing to identify mosaicism.

Finally, identification of a cohort of this size in a Japanese population raises further questions about the actual incidence of CCHS worldwide. With the 2010 ATS Statement on CCHS, the international figure of 1000 cases was presented (though presumed to be an underestimate). Ethnic disparity among reported individuals with CCHS is apparent, with Caucasians more heavily represented than any other ethnicity. ${ }^{6}$ Additionally, the incomplete penetrance and variable phenotype identified here and elsewhere in individuals with the 20/25 genotype indicates there may be a growing number of individuals with unrecognized mild hypoventilation who will necessitate clinical acumen and intellectual curiosity to identify. As knowledge of this disease spreads and utilization of $P H O X 2 B$ testing escalates, the perceived 'rareness' of CCHS may prove overstated.

1 Meguro, T., Yoshida, Y., Hayashi, M., Toyota, K. Otagiri, T., Mochizuki, N. et al. Inheritance of poly alanine expansion mutation of $P H O X 2 B$ in congenital central hypoventilation syndrome. J. Hum. Genet. 57, 335-337 (2012).

2 Matera, I., Bachetti, T., Puppo, F., Di Duca, M., Morandi, F., Casiraghi, G. M. et al. PHOX2B mutations and polyalanine expansions correlate with the severity of the respiratory phenotype and associated symptoms in both congenital and late onset Central Hypoventilation syndrome. J. Med. Genet. 41, 373-380 (2004).

3 Weese-Mayer, D. E., Berry-Kravis, E. M., Zhou, L., Maher, B. S., Silvestri, J. M., Curran, M. E. et al. Idiopathic congenital central hypoventilation syndrome: analysis of genes pertinent to early autonomic nervous system embryologic development and identification of mutations in PHOX2B. Am. J. Med. Genet. A. 123A, 267-278 (2003).

4 Bachetti, T., Parodi, S., Di Duca, M., Santamaria, G. Ravazzolo, R. \& Ceccherini, I. Low amounts of PHOX2B expanded alleles in asymptomatic parents suggest unsuspected recurrence risk in congenital central hypoventilation syndrome. J. Mol. Med. (Berl) 89, 505-513 (2011).

5 Jennings, L. J., Yu, M., Zhou, L., Rand, C. M. Berry-Kravis, E. M. \& Weese-Mayer, D. E. Comparison of $\mathrm{PHOX} 2 \mathrm{~B}$ testing methods in the diagnosis of congenital central hypoventilation syndrome and mosaic carriers. Diagn. Mol. Pathol. 19, 224-231 (2010).

6 Weese-Mayer, D. E., Berry-Kravis, E. M., Ceccherini, I., Keens, T. G. \& Trang, H. An official ATS clinical policy statement: congenital central hypoventilation syndrome: genetic basis, diagnosis, and management. Am. J. Respir. Crit. Care. Med. 181, 626-644 (2010).

7 Weese-Mayer, D. E., Patwari, P. P., Rand, C. M. Diedrich, A. M., Kuntz, N. L. \& Berry-Kravis, E. M. Congenital central hypoventilation syndrome (CCHS) and PHOX2B mutations. in Primer on the Autonomic Nervous System, 3rd edn (eds Robertson, D. Biaggioni, I., Burnstock, G., Low, P. A. \& Paton, J. F. R.) (San Diego, Academic Press, 2012). 